\title{
Erratum: Resonance Effects in Photoemission Time Delays [Phys. Rev. Lett. 115, 133001 (2015)]
}

\author{
M. Sabbar, S. Heuser, R. Boge, M. Lucchini, T. Carette, E. Lindroth, L. Gallmann, C. Cirelli, and U. Keller \\ (Received 15 August 2017; published 20 November 2017)
}

DOI: 10.1103/PhysRevLett.119.219901

In our Letter [1], we made a mistake in retrieving the delay curves from the calculated matrix elements. In addition we were unaware of a potential source of systematic errors in streaking measurements which we discussed in more details in [2]. In Fig. 1, we therefore can now present the corrected version of Fig. 3(b) of Ref. [1]. The multiconfigurational HartreeFock (MCHF) delay curve (magenta solid line) is retrieved from the calculated phases with the correct energy interval but neglecting the spectral width of the XUV and IR pulses.

The newly measured streaking delays (blue dashed line in Fig. 1) follow qualitatively the MCHF theory but significantly deviate from the theoretical preditiction of the random phase approximation with exchange (RPAE) model, which represents the Wigner delay without taking into account the Ar resonance (thin black line in Fig. 1). Additional measurements in [2] further confirm this difference. For this reason, the final conclusion of our original paper remains unchanged: in our measurements, we do not observe quantitative agreement between the experimental data and the theoretical Wigner delay calculated without taking into account the presence of resonances in argon.

Our new measurements do not reproduce the fast varying delays predicted by the MCHF calculations in the spectral region between 34 and $38 \mathrm{eV}$ (i.e. the magenta line in Fig. 1). We attribute this discrepancy to the finite energy resolution of the experiment, a question that should eventually be investigated by a proper account of the pulse widths in the theory curve; see e.g. [5].

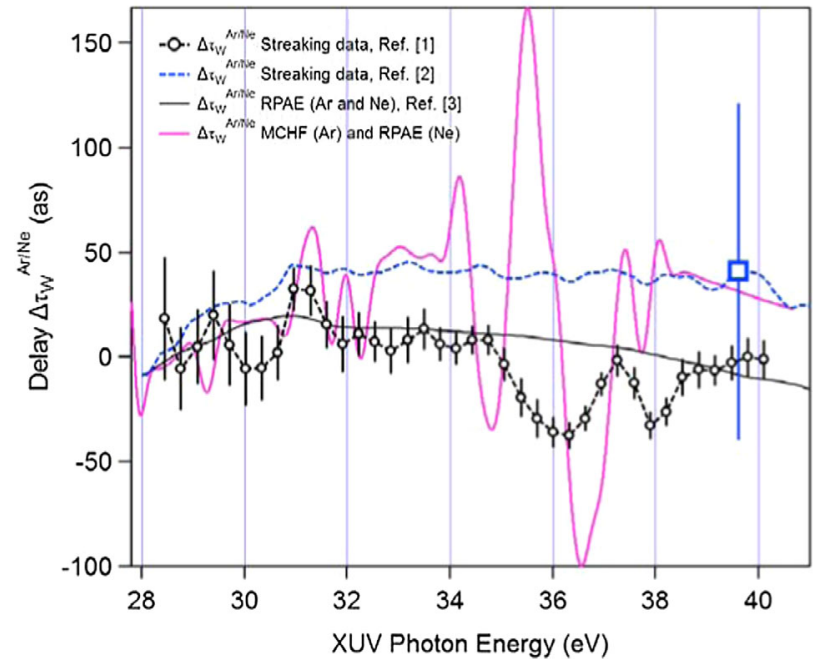

FIG. 1. Wigner time delay difference between Ar and Ne electrons as retrieved from attosecond streaking measurements. The black dashed line with open symbols represents the data published in Ref. [1], while the blue dashed line are the new data as published in Ref. [2]. In the latter data set, only the error bar of a single experimental point around $39.5 \mathrm{eV}$ is shown because the magnitude of the error bars is approximately the same for all the experimental data points between 28 and $41 \mathrm{eV}$. The experimental data are compared with two theoretical curves: the thin black line is computed within the RPAE both for Ar and Ne electrons [3]; the magenta one is obtained by using MCHF [4] for Ar and RPAE [3] for Ne and is calculated with an energy sampling of $0.3 \mathrm{eV}$. Only the MCHF is taking into account the Ar resonances in the continuum. 
[1] M. Sabbar, S. Heuser, R. Boge, M. Lucchini, T. Carette, E. Lindroth, L. Gallmann, C. Cirelli, and U. Keller, Phys. Rev. Lett. 115, 133001 (2015).

[2] L. Cattaneo, J. Vos, M. Lucchini, L. Gallmann, C. Cirelli, and U. Keller, Opt. Express 24, 29060 (2016).

[3] A. S. Kheifets, Phys. Rev. A 87, 063404 (2013).

[4] T. Carette, J. M. Dahlström, L. Argenti, and E. Lindroth, Phys. Rev. A 87, 023420 (2013).

[5] A. Jiménez-Galan, F. Martín, and L. Argenti, Phys. Rev. A 93, 023429 (2016). 